\title{
Research on optimized control for intelligent building adjustable resources
}

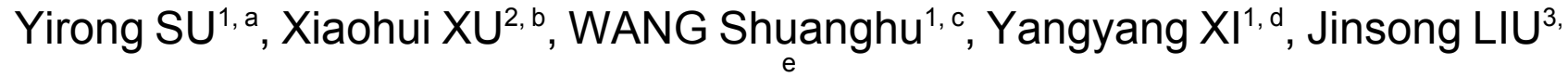 \\ ${ }^{1}$ State Grid Electric Power Research Institute, Nanjing, China \\ ${ }^{2}$ China Electric Power Research Institute, Renewable Energy Department, Nanjing, China \\ ${ }^{3}$ State Grid Shanghai Municipal Electric Power Company, Shanghai, China \\ aemail: syr@126.com, ${ }^{b}$ email: xuxiaohui@163.com, ${ }^{\mathrm{c} e m a i l:}$ wangshuanghu@163.com, ${ }^{\mathrm{d}} \mathrm{email}$ : \\ xyy@163.com, ${ }^{\mathrm{e} e m a i l:}$ jsl@163.com
}

Keywords: intelligent building, distributed generation, adjustable resources, load characteristic.

\begin{abstract}
In order to improve the smart grid energy efficiency and reduce the power cost, a kind of intelligent building is adjustable resource coordinate multi-objective optimization operation control model is presented through the analysis of intelligent building is adjustable resource load characteristic. The optimization goals contain smooth load fluctuation and electricity cost minimum. The multi-objective optimization is verified by calculation example the effectiveness of the operation control model of coordination, which both lowers the peak valley of the distribution network and decreases the cost building charging.
\end{abstract}

\section{Introduction}

Energy has become a symbol of human progress, from lighting, food, heating to cooling and so on that is inseparable from the energy. The energy shortage phenomenon has been ahead of the arrival ${ }^{[1]}$. In order to meet the needs of human energy consumption, the world's energy structure dominated by the oil develop to the multiple type, renewable energy also develop rapidly ${ }^{[2]}$.

Intelligent building is a micro power system that is made up of controllable load, Electric vehicles (Electric vehicle, EV), distributed power and optimal control protection and so on, which can be achieved with flexible distribution network interconnection or isolated island operation switch ${ }^{[3-5]}$. However, that the research on the micro power system in the intelligent building is relatively few in the aspects of adjustable resource optimal operation, energy demand management and so on.

A kind of intelligent building is adjustable resource coordinate multi-objective optimization operation control model that is designed in this paper, the main optimized goal of which is smoothing power grid load fluctuation and reducing electricity cost of building user. We construct the architecture of the optimal operation control and develop an optimal operation control strategy, to realize the operation effect of adjustable resource optimization in intelligent building, that is to both lower the peak- valley difference of distribution network and reduce the cost of building charging, which can provide reference for the optimization of electricity in intelligent building.

\section{Analysis of the optimal control model for intelligent building adjustable resource}

\section{The objective function}

Smooth the load fluctuation. To smooth the load fluctuation of grid is as the goal of the optimal operation of adjustable resource in intelligence building, the objective function of that is

$$
F_{1}=\min \sum_{t=1}^{T}\left(P_{L, t}+\sum_{i=1}^{n} P_{i, t}-\sum_{j=1}^{m} P_{j, t}\right)
$$


$T$ takes 24 , is the period throughout the day, $P_{L, t}$ is daily base load in the $t$ period of time, $n$ is the number of building distributed power, $P_{i, t}$ is the output power of the first $i$ distributed power in the first $t$ period, including the power of wind power, photovoltaic battery and electric vehicle discharge, etc, $m$ is the number of controllable load inside buildings, $P_{j, t}$ is the consumption of active power of the first $i$ controllable load in the first $t$ period.

The minimum cost of electricity. To get the minimum cost of electricity is as the goal of equipment in the intelligence building, the objective function is

$$
F_{2}=\min \sum_{t=1}^{T}\left(c_{t} \sum_{j=1}^{m} P_{j, t} \cdot t_{j}-\sum_{i=1}^{n}\left[c_{0} P_{i, t} \cdot t_{i}-\frac{C_{a i}}{24 P_{e i}} \cdot \frac{r(1+r)^{L_{i}}}{r(1+r)^{L_{i}}-1} \cdot P_{i, t}\right]\right)
$$

$c_{0}$ is the electricity price taken to $0.7 \mathrm{yuan} / \mathrm{kWh}, c_{t}$ is the time-sharing electricity price throughout the day, $t_{j}$ is the time of using electricity of the first $j$ controllable load in the first $t$ period, $C_{i, t}$ is the unit service time and maintenance cost of each distributed power, $C_{a i}$ is the installation cost per unit capacity of the first $i$ distributed power supply, $P_{e i}$ is the rated power of the first $i$ distributed power, unit of which is $\mathrm{kWh}, r$ is the discount rate, taking $8 \%$ in this article, $L_{i}$ is the service life of the first $i$ distributed power.

\section{Constraint condition}

Constraint of output power for distributed power.

$$
P_{i \min } \leq P_{i, t} \leq P_{i \max } \text { and } R_{i d} \cdot \Delta t \leq P_{i, t}-P_{i, t-1} \leq R_{i u} \cdot \Delta t
$$

$P_{i \min } 、 P_{i \max }$ are the minimum and maximum of the output power of the first $i$ distributed power respectively, $R_{i d} 、 R_{i u}$ are the first $i$ downward and upward ramp rate of distributed power.

Constraint of transmission power for micro grid system and distribution network.

$$
0 \leq P_{S, t} \leq P_{S \max } \text { and } 0 \leq P_{B, t} \leq P_{B \max }
$$

$P_{S \max }$ is the maximum active power, and $P_{B \max }$ is the maximum active power.

\section{Control strategy}

The optimization operation control architecture is shown in Fig.1.

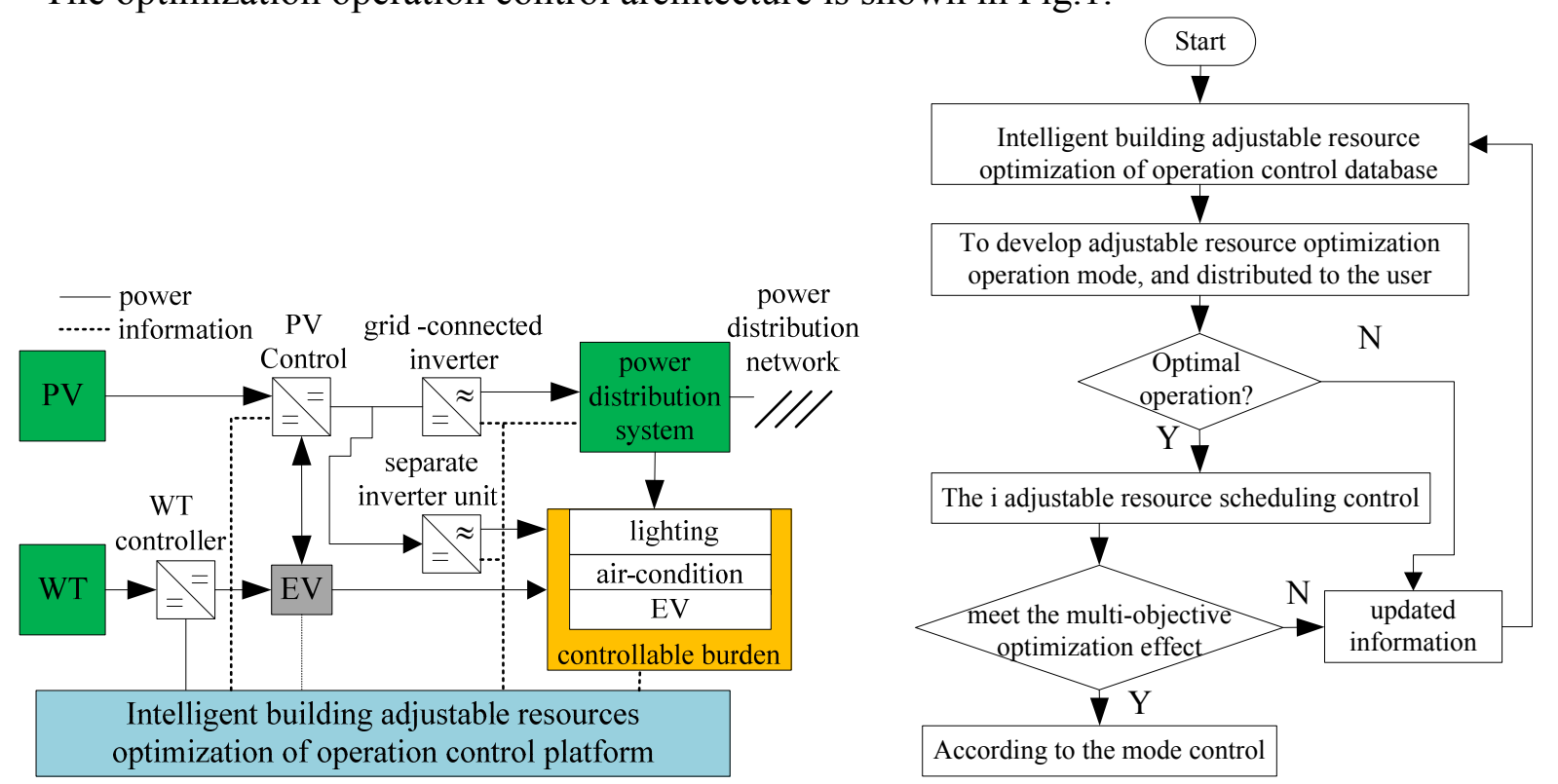

Fig.1 Optimization operation control architecture

Fig.2 Optimal operation control strategy

Through the online monitoring and scheduling function of the optimal operation control platform for intelligent building adjustable resource, to realize optimal operation control of adjustable resources including distributed power such as photovoltaic cell, wind power generation, etc, and controlled load like air conditioning, lighting, EV and so on. Among them, the EV both can be used as a controlled load and also used as a mobile energy storage unit. 
The multi-objective optimal control strategy for intelligent building is shown in Fig.2.Firstly, we need to establish information database about the optimized operation control for intelligent building adjustable resource, which contains all adjustable resources such as photovoltaic cells, wind power, EV, controllable load, etc. Then formulate optimization operation mode of adjustable resources, and send it to adjustable resources in the building. After the first i adjustable resource receiving a notice, which determines whether to allow the optimized operation mode, where if choosing no then update information database according to the constraint conditions, otherwise to judge whether meet the goal of multi-objective optimization, if meeting the goal then to continue to optimal operation based on this mode, otherwise to continue to update the database according to the constraint conditions.

\section{Example analysis}

In the example, optimization cycle is one day, divided into 24 hours, we use MATLAB software programming to realize. Assume that adjustable resources distribution in the building shown in table 1.

Table 1 Summary table of adjustable resources in the building

\begin{tabular}{|l|c|c|c|c|}
\hline Adjustable resource & $\begin{array}{l}\text { Quantit } \\
\mathrm{y}\end{array}$ & $P_{e i} / \mathrm{kW}$ & $C_{a i} /$ ten thousand yun & $L_{i} /$ year \\
\hline PV system & 5 sets & 8 & 1 & 15 \\
\hline WT system & 10 sets & 10 & 0.4 & 10 \\
\hline EV & 50 & 30 & 0.8 & 3 \\
\hline The air conditioning system & 60 sets & 5 & - & - \\
\hline Lighting equipment & 180 sets & 0.1 & - & - \\
\hline
\end{tabular}

In table 1, we only consider electricity cost of air conditioning and lighting without the installation cost and so on in this model, so the corresponding rated power, installation cost and service life are not given in this table. The daily base load curve of intelligence building is shown in figure 3 , where the output curve of photovoltaic and wind power are given according to table 1 . From the daily base load curve, we can know that the daily peak period mainly is 10 to 15 and 18 to 22 o'clock, daily maximum load appears around 20 o'clock, minimum load appears around 5 o'clock in the morning.
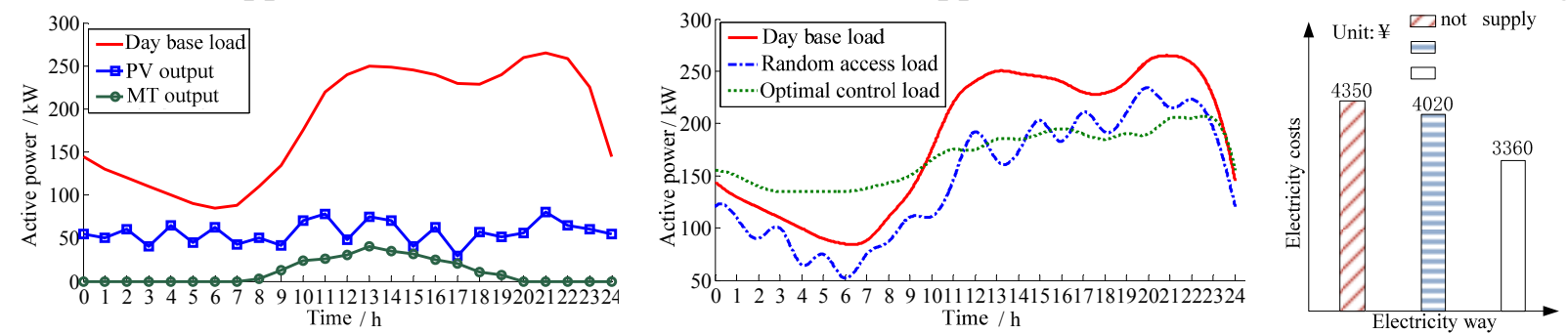

Fig.3 Output prediction curve

Fig.4 Daily load comparison curve Fig.5 cost comparison

Fig. 4 shows the comparison between the curve of power load in random participation and daily base load after the optimal operation control.

From the analysis of Fig. 4, it can be known that the overall load curve decline slightly after the random access of distributed power such as wind power and photovoltaic power, which have a serious disturbance to the power grid because wind power and photovoltaic power supply have the fluctuation. However, the fluctuation of load curve of output is greatly reduced after optimization control, and the peak and valley difference is also significantly reduced. The results show that the proposed model can achieve the purpose to smooth the load fluctuation, in addition, we can calculate the cost of electricity in the above three cases, which is shown in Fig. 5.

In Fig. 5, the cost of depreciation and maintenance increase due to the random access of the distributed power, where the cost of daily electricity only reduce by $330 ¥$, when we carry out the optimization control where the cost of daily electricity drop by $990 ¥$. 


\section{Summary}

This paper designs a optimization control model for intelligent building in which adjustable resource coordinate multi-objective operation, the goal of that is to smooth load fluctuation and reduce electricity cost in the building. In this paper, we construct the architecture of optimal operation control and formulate a strategy for optimal operation control through analyzing load characteristics and optimal control model of adjustable resource in the building to realize the effect of optimal operation of adjustable resource in the building that is both reducing peak and valley difference of distribution network in the building, and decreasing the cost of building charging. The model can provide reference for the optimizing electricity of the intelligent building.

\section{Acknowledgements}

This work was financially supported by China National science and technology support program projects funded projects (2013BAA01B04).

\section{References}

[1]Faisal A M, Heikki N K. Online management of micro-grid with battery storage using multio-bjective optimization, Powereng, 2007. Setubal (Portugal), 2007: 231-236.

[2] HE Yongxiu, WANG Bing, XIONG Wei, et al. Analysis of residents' smart electricity consumption behavior based on fuzzy synthetic evaluation and the design of interactive mechanism, Power System Technology, 2012, 36(10), pp.247-252.

[3] HUANG Li, WEI Zhinong, WEI YanfangXU Fan, et al. A survey on interactive system and operation patterns of intelligent power utilization, A Survey on Interactive System and Operation Patterns of Intelligent Power Utilization, 2013, 37(8), pp.2230-2237.

[4] ZHU bingruo, ZHANG shenming, WANG hu. Realization means of integrated energy-saving control in intelligent power building, Shanghai Energy Conservation, 2010, 4: 39-43.

[5] CHEN Liangliang, ZHANG Beibei, ZHOU Bin, et al, A Time-of-use price based multi-objective optimal dispatching for charging and discharging of electric vehicles, Power System Technology, 2014, 38(11), pp.2972-2977. 